\title{
Super Efficiency Evaluation using a Common Platform on a Cooperative Game
}

\author{
Yongjun $\mathbf{L i}^{* 1}$ \\ Email: lionli@ustc.edu.cn \\ Jianhui Xie ${ }^{1}$ \\ Email: jeff@mail.ustc.edu.cn \\ Meiqiang Wang ${ }^{2}$ \\ E-mail: wangmq@mail.ustc.edu.cn \\ Liang Liang ${ }^{1}$ \\ Email: lliang@ustc.edu.cn \\ ${ }^{1}$ School of Management, University of Science and Technology of China \\ Hefei, P.R. China 230026 \\ ${ }^{2}$ School of Management, Guizhou University \\ Guiyang, P.R. China 550025
}

\begin{abstract}
Classical DEA models (such as CCR and BCC models) compute efficiencies of decisionmaking units (DMUs) based on a common efficient frontier but with no capability of differentiating efficient units. The super efficiency model was developed to rank efficient DMUs based a new efficient frontier comprised by all other DMUs. This may lead to a multi-platform problem that different efficient DMUs are evaluated based on different efficient frontiers and the resulted super efficiencies of efficient DMUs are not comparable. This paper addresses the multi-platform problem from the perspective of a cooperative game. Efficient DMUs are regarded as players and subsets of these efficient DMUs as coalitions. The effect of a coalition on a specific efficient DMU is defined as the DMU's efficiency change proportion (ECP) based on the traditional DEA models when the coalition is removed from the reference set. Basing on the ECP, we define a characteristic function as the sum of all efficient DMUs'ECPs, and prove that this function is super-additive. Then, the Shapley value is introduced as a solution of this cooperative game and applied to rank efficient DMUs. The proposed approach is demonstrated by two numerical examples. Finally, we extend the proposed approach in this paper to the VRS assumption.
\end{abstract}

Key words: Data envelopment analysis (DEA); Super efficiency; Common platform; Cooperative game; Efficiency change proportion (ECP).

\footnotetext{
${ }^{*}$ Corresponding author.
} 


\section{Introduction}

Originating from the work of Farrell (1957) and Debreu (1951), data envelopment analysis (DEA) developed by Charnes, Cooper, \& Rhodes (1978) is a popular nonparametric method to evaluate performance of various peer decision-making units (DMUs). Now, DEA has been widely applied to measuring performance in many areas such as universities (Lim \& Zhu, 2013), banks (Staub, Souza, \& Tabak, 2010; Wanke, Barros, \& Emrouznejad, 2016), reallocation of emission permits (Wu, Du, Liang, \& Zhou, 2013), and health care (Pulina, Detotto, \& Paba, 2010; Shwartza, Burgess, \& Zhu, 2016). Its popularity can be attributed to no requirements specific production functional form and piror knowledge of DMUs' input and output weights (Cooper, Seiford, \& Tone, 2007; Cooper, Seiford, \& Zhu, 2011). In our opinion, another reason of its popularity is that DMUs are evaluated fairly under a common frontier comprised of all DMUs, and such a frontier constitutes a common platform for all DMUs. Therefore, efficiencies obtained by classical DEA models are comparable and can be used to rank DMUs. However, classical DEA models fail to rank (weakly) efficient DMUs ${ }^{1}$, for all efficient DMUs' efficiencies are ones.

A number of novel approaches are proposed to rank efficient DMUs (Angulo \& Lins, 2002; Adler, Friedman, \& Stern, 2002). For example, super efficiency, introduced by Peterson and Anderson (1993), is one of the important approaches for ranking efficient DMUs. In this approach, each efficient DMU is evaluated by an efficient frontier comprised by all other DMUs. If a DMU is strongly efficient, it may be beyond of envelopment of such the frontier. Therefore, the efficient DMU's super efficiency may be bigger than one. In this case, super efficiency is a popular approach used to rank efficient DMUs. And it has been widely applied in many areas such as fixed cost allocation (Li, Yang, Liang, \& Hua, 2009), manufacturing firm evaluation (Düzakın \& Düzakın, 2007), and bank evaluation (Avkiran, 2011). However, this model brings at least two problems. The first is that the model may be infeasible based on the variable return to scale (VRS) assumption (Baker \& Talluri, 1997; Seiford \& Zhu, 1999; Cook, Liang, Zha, \& Zhu, 2008; Johnson \& McGinnis, 2009; Lee, Chu, \& Zhu, 2011; Chen \& Liang, 2011; Lee \& Zhu, 2012; Chen, 2013). The second is the multi-platform problem. Super efficiency model evaluates each efficient DMU by a specified efficient frontier comprised of other DMUs, and such the frontier may vary when different efficient DMUs are evaluated. Therefore, super efficiency evaluates these efficient DMUs based on different standards,

\footnotetext{
${ }^{1}$ In this paper, "efficient DMUs" refer to DMUs that are on the efficient frontier. Therefore, for "radial" DEA models, the set of "efficient DMUs" not only includes strongly Pareto-efficient DMUs but also weakly efficient DMUs. For non-radial DEA models, such as the slack-based measure model, "efficient DMUs" only refer to Pareto-efficient DMUs.
} 
and super efficiencies of efficient DMUs may be not comparable. For details, readers can refer to Section 3. Similarly, Banker \& Chang (2006) argued that the super efficiency model is useful for outlier identification but not good at ranking efficient units. Banker, Chang, \& Zheng (2015) found that the reason of unsatisfactorily comes from the "left corner" of DMUs.

Based on the philosophy of super efficiency, a novel approach of changing the reference set is proposed to discriminate efficient DMUs (Chen \& Deng, 2011; Jahanshahloo, Junior, Lotfi, \& Akbarian, 2007; Hibiki \& Sueyoshi, 1999). This approach regards each efficient DMU as a candidate. For a given candidate, its effect is defined as the sum of the efficiency changes of other DMUs when the candidate is omitted from the reference set. Such an effect is called the DEA cross reference (DCR). This approach ranks each efficient DMU based on its DCR value. The higher its DCR value, the higher its rank. However, the nature of this approach is the same as the super efficiency, and it still has the multi-platform problem.

Cross-efficiency is another important approach of ranking efficient DMUs. It is first proposed by Doyle \& Green (1994) and developed by Liang Wu, Cook, \& Zhu (2008), Wu, Liang, \& Yang (2009), Wu, Liang, \& Chen (2009), Wu, Liang, Yang, \& Yan, (2009), Alcaraz, Ramón, \& Ruiz (2013), Ruiz (2013) and Wu, Chu, Sun, \& Zhu, (2016). This approach allows each DMU to selfishly choose an optimal set of input and output weights to the classical DEA, and defines the average value of a DMU efficiencies based on these optimal weights as its cross efficiency. However, cross-efficiency scores are generally not unique, and depend on which of the alternate optimal solutions to the DEA linear programs is used (Liang, Wu, Cook, \& Zhu, 2008).

Common weight DEA is also an approach for ranking DMUs (Cook, Roll, \& Kazakov, 1990; Roll, Cook, \& Golany, 1991; Kao \& Hung, 2005; Wu, Liang, \& Yang, 2009; Kao, 2010; Wu, Chu, Zhu, Li, \& Liang, 2016). This approach attempts to find a common set of weights to calculate efficiencies for all DMUs, and ranks them based on their efficiencies. Compared to the cross efficiency, this approach evaluates DMUs based on a common platform (a common set of weight), and the obtained efficiencies are comparable. However, it's difficult to find a comprise principle to choose the common set of weights and different principles adopted lead to different common sets of weights chosen as well as ranks among DMUs.

Besides, Salo \& Punkka (2011) introduced the efficiency dominance to rank DMUs by considering all possible input and output weights: a DMU is only said to be better than another DMU if its efficiency score is higher for all feasible input and output weights. This approach is fair to rank DMUs but lacks of discrimination for efficient DMUs. 
In the current study, we approach the multi-platform problem in efficiency evaluation from the perspective of cooperative game theory. Efficient DMUs are assumed to compete and cooperate with each other in the evaluation. For example, in bank performance evaluation, efficient branches compete with other branches to be the best benchmark because branches and their managers with higher ranks often receive more rewards and support in the future. On the contrary, efficient branches cooperate with each other to construct a common efficient frontier to evaluate inefficient ones, and make the evaluate result comparable.

Combining DEA with game theory is not rare in DEA literature, Nakabayashi \& Tone (2006) presented a cooperative game to solve the problem of consensus-making between unit and organization when evaluating performance via DEA models. Li \& Liang (2010) used a cooperative game to select indices for DEA. Some researches focused on incorporating game theory with a twostage DEA model (Zhu, 2004; Liang, Cook, \& Zhu, 2008; Zha \& Liang, 2010; Du Liang Chen Cook \& Zhu, 2011; Li, Chen, Liang, \& Xie, 2012; Zhou, Sun, Yang, Liu, \& Ma, 2013). Lozano (2012; 2013), Lozano, Mármol, \& Hinojosa (2015), Borrero, Hinojosa, \& Mármol (2016) and Wu, Zhu, Cook, \& Zhu (2016) proposed approaches based on cooperative games in dealing with problems of information and technology sharing among organizations.

The purpose of this paper is to rank efficient DMUs with a common platform. We define a cooperative game with transferable utility (TU-game) to construct the common platform for efficient DMUs. Each efficient DMU is regarded as a player, each possible subset of efficient DMUs is regarded as a coalition. The effect of a coalition on each efficient DMU is defined as its efficiency change proportion (ECP) when the coalition is removed from the reference set. Basing on ECP, we define a characteristic function of the coalition as the sum of the ECPs of all efficient DMUs. We also prove that the characteristic function satisfies super additivity. Then, with the fairness axioms of efficiency and dummy player, symmetry and additivity, a Shapley value is used as a solution to the cooperative game and to rank DMUs. In this paper, we will also extend the proposed approach under the variable returns to scale (VRS) assumption.

The remainder of this paper is organized as follows. Section 2 describes the problem; Section 3 defines ECP to measure the marginal role of a DMU set on an efficient unit; Section 4 presents a common platform approach based TU-game for ranking efficient DMUs; Numerical examples are presented in Section 5; Section 6 extends our common platform approach to the VRS assumption; The conclusion is elaborated in Section 7.

\section{Problem description}


In this paper, we use the following notations:

Table 1 Notation explanation

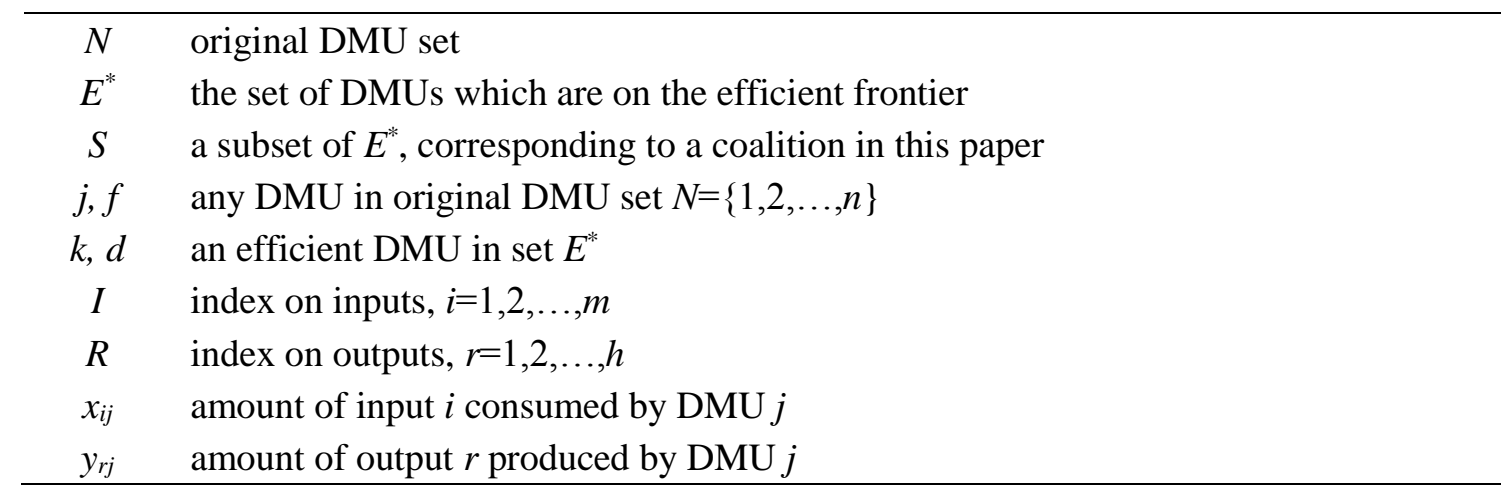

Notably, $E^{*}$ defined in Table 1 is the set of DMUs that are on the efficient frontier. Therefore, their efficiencies are all 1. For the "radial" DEA models in this section, $E^{*}$ may not only include strongly efficient DMUs, but also include weakly efficient DMUs. Suppose there are $n$ independent DMUs in set $N$, and each $D M U_{j}(j \in N=\{1,2, \ldots, n\})$ consumes $m$ inputs $x_{i j}(i \in M=\{1,2, \ldots, m\})$ to generate $h$ outputs $y_{r j}(r \in H=\{1,2, \ldots, h\})$. The efficiency rating for any given $D M U_{f}$ can be computed by using the standard input-oriented CCR model (Charnes, Cooper, \& Rhodes, 1978) as follows:

$$
\begin{array}{ll} 
& E_{f}(N)=\operatorname{Min} \theta \\
\text { s.t. } & \sum_{j \in N} \lambda_{j} x_{i j} \leq \theta x_{i f}, i \in M \\
& \sum_{j \in N} \lambda_{j} y_{r j} \geq y_{r f}, r \in H \\
& \lambda_{j} \geq 0, \forall f, j \in N, \theta: \text { free }
\end{array}
$$

where $E_{f}(N)$ is the efficiency for $D M U_{f}$ based on reference set $N$.

The super efficiency model (Peterson and Anderson, 1993) for evaluating efficient units can be formulated as follows:

$$
\begin{array}{ll} 
& E_{f}(N)=\operatorname{Min} \theta \\
\text { s.t. } & \sum_{\substack{j \in N \\
j \neq f}} \lambda_{j} x_{i j} \leq \theta x_{i f}, i \in M \\
& \sum_{\substack{j \in N \\
j \neq f}} \lambda_{j} y_{r j} \geq y_{r f}, r \in H \\
& \lambda_{j} \geq 0, \forall f, j \in N, j \neq f, \theta: \text { free }
\end{array}
$$


If DMU $f$ is an efficient DMU, then its efficiency computed by the super efficiency model is not less than 1. While if DMU $f$ is an inefficient DMU, then its super efficiency score equals to its CCR score.

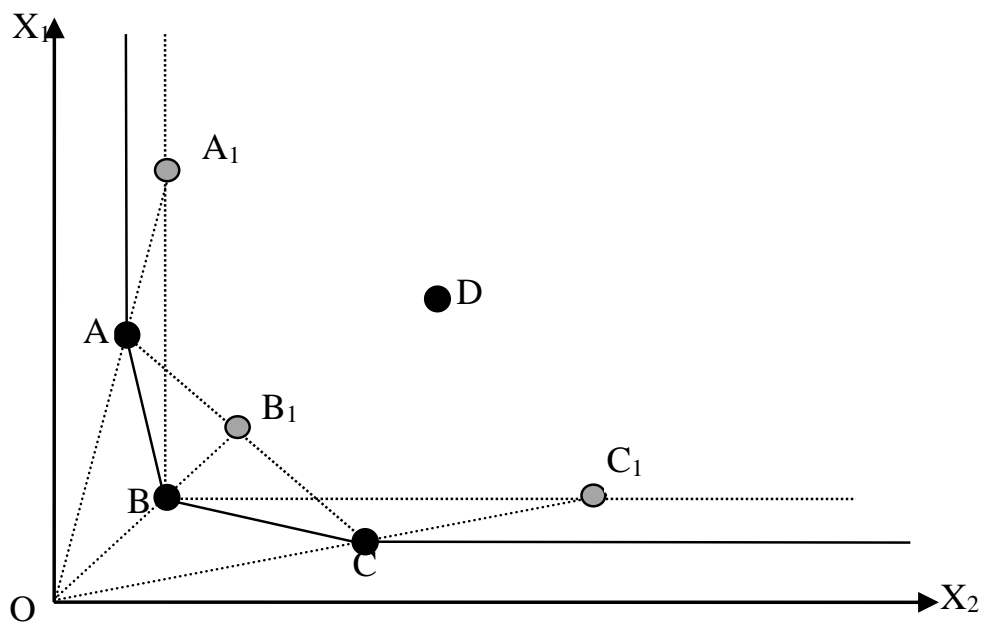

Figure 1 Efficient frontiers formed by different efficient DMUs

We use the example described in Figure 1 to explain the multi-platform problem in detail. Figure 1 plots four DMUs: $A, B, C$, and $D$. Each DMU has two inputs $\mathrm{X}_{1}$ and $\mathrm{X}_{2}$ with a single output of unity. Three efficient DMUs $A, B$, and $C$ and one inefficient DMU $D$ are also shown. If the super efficiency model is used to evaluate $A$, the efficient frontier is $A_{1} B C$ and the super efficiency score of $A$ is calculated as $O A / O A_{l}$. In a similar manner, $B$ 's super efficiency score is $O B / O B_{1}$ and the efficient frontier is $A B_{1} C$. The reference set of $C$ is $A B C_{l}$ when $C$ is under evaluation and its super efficiency score is $O C / O C_{1}$. Notably, the efficient frontiers based on the super efficiency model are changing when different efficient DMUs are evaluated. Three different efficient frontiers are used when evaluating super efficiencies of $A, B$, and $C$, respectively. These three efficient frontiers imply that $A$, $B$, and $C$ are not evaluated with the same evaluation standards. Hence, super efficiencies of $A, B$, and $C$ are not comparable.

Meanwhile, if we evaluate all efficient DMUs based on one single frontier (the same reference set), then resulted efficiencies may not be fair. For example, denote the set of removed efficient DMUs as $S$ and the set of all DMUs as $N$. When $S$ is removed, the new reference set is denoted as $N / S$. The efficiency of DMU $k$ can be computed by model (3) as follows:

$$
\begin{array}{ll} 
& E_{k}(N / S)=\operatorname{Min} \quad \theta \\
\text { s.t. } & \sum_{j \in N / S} \lambda_{j} x_{i j} \leq \theta x_{i k}, i \in M \\
& \sum_{j \in N / S} \lambda_{j} y_{r j} \geq y_{r k}, r \in H \\
& \lambda_{j} \geq 0, \forall k \in E^{*}, j \in N / S
\end{array}
$$


Using Figure 1 as an example, if $S=\{A\}$, then the rest of efficient DMUs set $N\{A\}$ forms an efficient frontier $A_{l} B C$. Based on this frontier, DMU $A$ 's score calculated by model (3) is higher than 1, whereas DMU B's score equals to 1 . The scores are comparable for the DMUs based on one common efficient frontier $A_{1} B C$, but unfair for DMU $B$ for only considering the subset $S=\{A\}$. On the contrary, the scores would be comparable but the situation would be unfair for DMU $A$ if we only consider the subset $S=\{B\}$. The reason is that when we set $S=\{B\}$, the resulted frontier $A B_{1} C$ would be of advantage to DMU $B$ 's supper efficiency, whereas be of disadvantage to DMU A's super efficiency.

Therefore, a conflict arises, that is, if we use the super efficiency model, efficient DMUs can be discriminated while super efficiencies resulted may be inequitable or incomparable. If we use CCR model, the resulted efficiencies are equitable and comparable, while efficient DMUs can't be discriminated.

\section{Marginal effect of an efficient DMU set on an efficient unit}

In this section, we define a new measure, which quantifies the marginal impact of a particular DMU set on an efficient unit in efficiency evaluation. This particular DMU set should be a subset of $E^{*}$.

Definition 1: The effect of an efficient DMU set $S$ on an efficient $D M U_{d}$ is defined as $E C P_{d}(S)$ :

$$
E C P_{d}(S)=\frac{E_{d}(N / S)}{E_{d}(N)}-1=E_{d}(N / S)-1, \forall d \in E^{*}, S \subseteq E^{*},
$$

where $E_{d}(N / S)$ is the efficiency score of $D M U_{d}$ computed by model (3) with the DMU set $N / S$ and $E_{d}(N)=1$ is computed by model (1). Obviously, $\forall d \in E^{*}, S \subseteq E^{*}$, if $d \notin S$, then $E C P_{d}(S)=0$; if $d \in S$, then $E C P_{d}(S) \geq 0$; if $S=\varnothing$, then $E C P_{d}(S)=0$.

When we remove an efficient DMU set $S$ from the reference set, efficient DMUs outside $S$ are still on the frontier, therefore, if $d \notin S$, then $E_{d}(S)=0$. While if $S=\varnothing$, which means every efficient DMU is outside $S$, then we also have $\operatorname{ECP}_{d}(S)=0$.

Consider the effect of removing a DMU in $S$. When $S$ contains only one DMU, our definition that characterizes the effect of deleting an efficient DMU does exactly what the super efficiency model does. In the super efficiency model, each efficient DMU is measured by a new efficient frontier composed by the efficient DMU set without the targeted one. Based on the super efficiency model, the efficiency score of each efficient DMU may be higher than 1. For example, we suppose an 
efficient DMU $d$ has a super efficiency score of 1.2 contrary to its CCR score of 1 . Such a difference between the two scores is attributed to the two different efficient frontiers used in the different models. The super efficiency model forms a new efficient frontier by removing the DMU under consideration from the DMU set, whereas the CCR model forms an efficient frontier composed of all efficient DMUs including the DMU under consideration. When $S$ has more than one DMU, if $d \in S$, then the efficient DMU $d$ is evaluated by a new efficient frontier composed of the DMU set when the DMUs in $S$ have been removed. The score $E_{d}(S)$ is higher than or equivalent to 0 . In this case, our definition also characterizes such an effect of removing an efficient DMU set.

The definition of $E C P_{d}(S)$ captures the idea that the proportion of the efficiency score of DMU $d$ will change when coalition $S$ is removed from reference set. In other words, the value $E C P_{d}(S)$ illustrates the extent of the effect of coalition $S$ on DMU $d$. Based on Definition 1, if we obtain $E C P_{d}(S)$, then the efficiency of DMU $d$ against the new efficient frontier enveloped by the DMU set $N / S$, namely, $E_{d}(N / S)$, can be easily computed. Previously, to ensure comparability and equitability in the evaluation, we need to consider all possible efficient frontiers comprised of different efficient DMU sets.

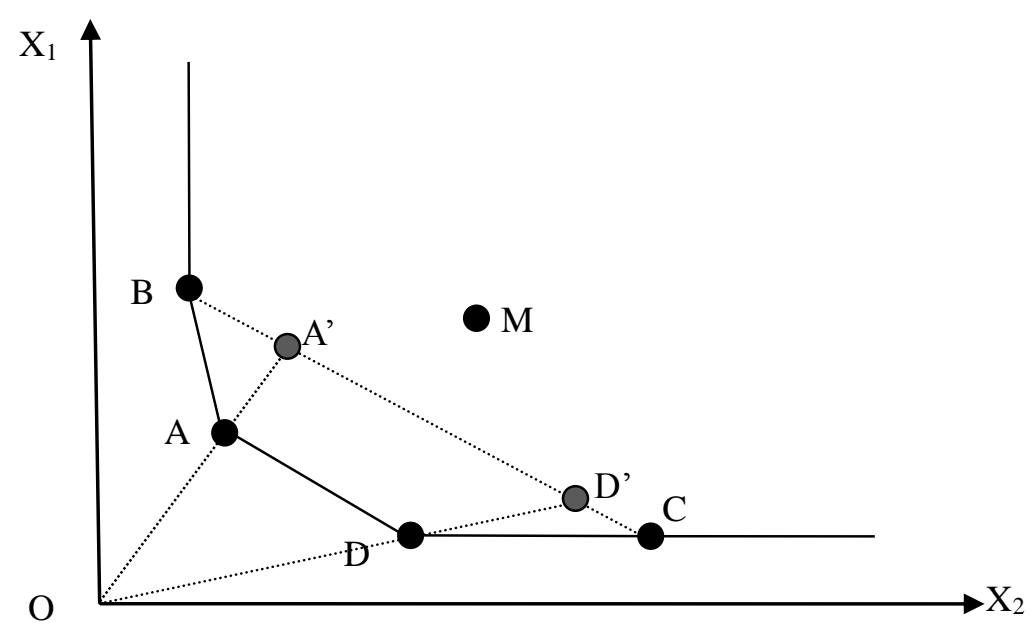

Figure 2 ECP of each DMU

We use Figure 2 to illustrate how to calculate $\operatorname{ECP}_{d}(S)$ and clarify the concept of ECP. The figure shows five DMUs: $A, B, C, D$, and $M$. Each DMU consumes two inputs, $\mathrm{X}_{1}$ and $\mathrm{X}_{2}$ to generate a unit of output. The figure also shows that $A, B, C$, and $D$ are efficient DMUs in the CCR model while $M$ is an inefficient DMU. If $S=\{A, D\}$ and $D$ is the targeted DMU, then $E C P_{D}(S)=\frac{E C P_{D}(N / S)}{E C P_{D}(N)}-1=E C P_{D}(N / S)-1=\frac{O D^{\prime} / O D}{O D / O D}-1=\frac{D D^{\prime}}{O D} . E C P_{D}(S)$ means $D^{\prime}$ 's ECP 
when DMU set $S$ is removed from the reference set. Similarly, $E C P_{A}(S)=\frac{E C P_{A}(N / S)}{E C P_{A}(N)}-1=E C P_{A}(N / S)-1=\frac{O A^{\prime} / O A}{O A / O A}-1=\frac{A A^{\prime}}{O A}$.

\section{Cooperative game in determining the rank of each efficient DMU}

In this section, we assume that efficient DMUs cooperate with each other to maintain a common efficient frontier. Evaluation results based on the common efficient frontier are comparable and equitable. We use a cooperative game to construct a common platform and consider the competitive and cooperative relationships among efficient DMUs in $E^{*}$. Each efficient DMU is regarded as a player, $E^{*}$ is regarded as a grand coalition.

Definition 2: A common platform cooperative game with transferable utility (TU-game) can be denoted as $\left\langle E^{*}, C\right\rangle$, which consists of

1) a finite set $E^{*}$, the set of players (efficient DMUs)

2) a function $C$ assigned to every subset (coalition) $S$ of $E^{*}$, that is, $\forall S \subseteq E^{*}$, a real number $C(S)$ exists. $C: 2^{E^{*}} \rightarrow R$ is denoted as the characteristic function, and $C(S)$ is the total payoff of coalition $S$. The coalition $E^{*}$ can be called as grand coalition.

Definition 3: For a given coalition $S$, the value of corresponding payoff $C(S)$ is defined as

$$
C(S)=\sum_{d \in S} E C P_{d}(S), \forall S \subseteq E^{*}
$$

where $S$ is a subset of efficient unit set $E^{*} . C(S)$ is available for all possible divisions among the players of $S$, and $C(\varnothing)=0$. The definition means that the higher the effect of a coalition $S$ on the efficient DMUs, the higher the payoff of $S$.

Given that $E C P_{d}(S)$ measures the effect of coalition $S$ on DMU $d, C(S)$ is the effect of coalition $S$ on the original efficient frontier. In other words, $C(S)$ captures the concept of how much the efficient frontier will change when the coalition $S$ does not participate in the evaluation. In this study, $C(S)$ is also defined as the overall payoff of coalition $S$ in the TU-game. Therefore, $C(S)$ should be divided among players of $S$.

We evaluate the performance of bank branches as an example. In the first step, the traditional DEA models are used to classify efficient branches and non-efficient branches. Then, in the second 
step, a cooperative game is used for evaluating efficient branches. In this study, rewards are regarded as the payoff, and we assume that players drive a same utility from the same amount of rewards. Efficient branches cooperate with each other to obtain a higher total rewards (payoff, $C(S)$ ). The total rewards $C(S)$ can also be allocated to each efficient branch with fairness in the TU-game.

Theorem 1: The characteristic function $C$ is super-additive. Specifically, for any $S_{1} \subseteq E^{*}$ and $S_{2} \subseteq E^{*}$ with $S_{1} \cap S_{2}=\varnothing$, we obtain

$$
C\left(S_{1} \cup S_{2}\right) \geq C\left(S_{1}\right)+C\left(S_{2}\right) .
$$

Proof. See Appendix.

We seek a fair mechanism to allocate the expected overall gains among efficient DMUs in the coalition. First, following Osborne \& Rubinstein (1994), we introduce three axioms to formalize notions of fairness as follows.

1), Efficiency and Dummy Player: All the gains generated by the grand coalition should be allocated. If a DMU $i$ does not make any contribution to any of the coalitions it joins, that is, $\operatorname{ECP}_{i}(S)=0$ for all $S$ such that $i \in S$, then the allocated value for DMU $i$ is zero..

2), Symmetry: We set an interchange function $\pi: E^{*} \rightarrow E^{*}$, which changes the index of DMU from $i$ to $\pi(i)$. Then, we have that the allocation for $i$ equals to $\pi(i)$.

3), Additivity: For any two games $C_{1}$ and $C_{2}$, we have that the allocation for $C_{1}+C_{2}$ is equal to the sum of allocation for $C_{1}$ and $C_{2}$.

Notably, $k_{1}$ and $k_{2}$ are interchangeable in axiom Symmetry and are defined as: $\sum_{d \in s \cup\left\{k_{1}\right\}} E C P_{d}\left(S \cup\left\{k_{1}\right\}\right)=\sum_{d \in S \cup\left\{k_{2}\right\}} E C P_{d}\left(S \cup\left\{k_{2}\right\}\right)$ for any $S$ that contains neither $k_{1}$ nor $k_{2}$. In this axiom, if $k_{1}$ and $k_{2}$ are interchangeable, then they have equal contribution for any possible coalitions in $E^{*}$. Therefore, they should also be allocated with an equal value from the coalition. For the super efficiency common platform TU-game, if DMU $k_{1}$ and DMU $k_{2}$ have same inputs and outputs, then they should also have a same ranking.

As discussed in Shapley (1953) and Myerson (1991), Shapley value is the unique allocation that satisfies the above axioms, and can be computed by the following Formula (6): 


$$
\varphi_{k}(C)=\sum_{k \in S, S \subseteq E^{*}} \frac{(p-1) !(q-p) !}{q !}[C(S)-C(S /\{k\})],
$$

where $p$ is the number of units in DMU set $S$, i.e. $p=|S|$, and $q$ is the number of units in $E^{*}$ i.e. $q=\left|E^{*}\right| . S$ can be any possible subset of efficient DMU set $E^{*}$.

Definition 3 shows that $S$ cannot be equivalent to $E^{*}$ when no inefficient unit exists in $N$. Since the Shapley value method needs to compute $C(S)$, when all efficient DMUs are omitted. If at least one inefficient DMU exists, then the Shapley value for each efficient DMU can be calculated; if no inefficient DMU exists in the DMU set, then the efficient frontier does not exist when we omit all efficient units. In this case, a new DMU needs to be formed. In our approach, we create a new DMU as $X_{i}^{-}=\max _{k \in E^{*}}\left\{X_{i k}\right\}, i=1, \ldots, m$ and $Y_{r}^{-}=\max _{k \in E^{*}}\left\{Y_{r k}\right\}, r=1, \ldots, s$. Such a new unit is denoted as $E^{-}$. After that, we can calculate the Shapley value for each efficient DMU based on Formula (6).

\section{Numerical examples}

In this section, we illustrate the proposed approach using two data sets. One is an illustrative data, and the other is a real-world data of 14 Chinese city commercial banks (CCCBs) in 2012. In the two examples, we compute the Shapley value and rank of each efficient DMU and compare the evaluation results with the super efficiency model.

\subsection{Illustrative data example}

The illustrative data set contains eight DMUs, as shown in Table 2. Each DMU consumes two inputs: $X_{1}$ and $X_{2}$, to generate two outputs: $Y_{1}$ and $Y_{2}$. Our approach and the super efficiency model are applied to this data set. The evaluation results are shown in Table 3.

Table 2 Illustrative data set

\begin{tabular}{ccccc}
\hline & Input $\mathrm{X}_{1}$ & Input $\mathrm{X}_{2}$ & Output $\mathrm{Y}_{1}$ & Output $\mathrm{Y}_{2}$ \\
\hline$A$ & 2 & 7 & 3 & 1 \\
$B$ & 2 & 12 & 4 & 1 \\
$C$ & 5 & 5 & 2 & 1 \\
$D$ & 10 & 4 & 2 & 1 \\
$M$ & 3 & 6 & 1 & 1 \\
$F$ & 10 & 6 & 1 & 1 \\
\hline
\end{tabular}




\begin{tabular}{lllll}
\hline$G$ & 4 & 12 & 2.5 & 1 \\
$H$ & 5 & 11 & 3.5 & 1 \\
\hline
\end{tabular}

Based on the CCR model, DMUs $A, B, C, D$, and $M$ are efficient, whereas DMUs $F$, $G$, and $H$ are inefficient. Therefore, the main assignment of the evaluation is to compare the five efficient DMUs. Table 3 shows the efficiency and rank of each efficient unit based on the super efficiency model and our common platform approach. Comparing the $2^{\text {nd }}$ and $4^{\text {th }}$ columns, we can find that the ranks of unit $C$ and unit $D$ are exchanged.

Table 3 Results of CCR model

\begin{tabular}{cccccc}
\hline Unit & Super efficiency & Rank (SE) & Shapley value & Rank & Rank change \\
\hline$A$ & 1.3582 & 1 & 2.1517 & 1 & 0 \\
$B$ & 1.3333 & 2 & 1.5705 & 2 & 0 \\
$C$ & 1.1000 & 4 & 0.7827 & 3 & +1 \\
$D$ & 1.2500 & 3 & 0.6753 & 4 & -1 \\
$M$ & 1.0417 & 5 & 0.3881 & 5 & 0 \\
\hline
\end{tabular}

\subsection{Real-world example}

Our proposal is also applied to the data set of 14 CCCBs in 2012. The data is from each bank's annual report. Each bank has three inputs and two outputs, as shown in Table 4. The inputs include Fixed assets, Employee's pay and General expenses. The outputs include Profit and Loans. The inputs and outputs are measured in thousand Yuan.

Table 4 Data set of Chinese commercial banks of 2012

\begin{tabular}{lccccc}
\hline Bank & Fixed assets & Employees' pay & General expenses & Profit & Loans \\
\hline Jiujiang Bank $A$ & 775,310 & 283,893 & 644,280 & $2,193,085$ & $26,392,924$ \\
Chengdu Bank & $1,120,795$ & 679,087 & $1,674,877$ & $3,262,998$ & $91,799,733$ \\
Baotou Bank & $5,086,018$ & 420,404 & $2,867,703$ & $2,845,104$ & $57,320,923$ \\
Hangzhou Bank & $1,114,333$ & $1,239,207$ & $3,187,168$ & $4,474,572$ & $148,341,428$ \\
Huishang Bank $C$ & $1,178,797$ & $1,155,569$ & $2,433,518$ & $5,680,038$ & $159,941,475$ \\
Harbin Bank & $5,810,688$ & 332,044 & $2,466,586$ & $3,789,997$ & $85,298,079$ \\
Jiangsu Bank & $3,203,353$ & $2,864,359$ & $6,617,403$ & $9,625,877$ & $342,827,271$ \\
ZHHR Bank $F$ & 150,570 & 207,802 & 877,996 & 636,046 & $21,152,061$
\end{tabular}




\begin{tabular}{|c|c|c|c|c|c|}
\hline Tianjin Bank $D$ & $1,267,597$ & 296,940 & $1,820,934$ & $3,298,603$ & $118,767,291$ \\
\hline Chongqing Bank $E$ & $1,015,688$ & 142,024 & $1,577,106$ & $2,518,447$ & $75,256,873$ \\
\hline Dalian Bank & $1,154,635$ & 619,940 & $2,327,875$ & $2,253,330$ & $98,880,747$ \\
\hline Hankou Bank $B$ & $1,088,335$ & 95,372 & $1,221,129$ & $2,414,775$ & $57,757,208$ \\
\hline Nanjing Bank & $2,172,992$ & 635,475 & $2,721,211$ & $4,980,404$ & $121,962,186$ \\
\hline Ningbo Bank & $2,397,820$ & 624,031 & $3,529,395$ & $5,098,041$ & $142,564,629$ \\
\hline
\end{tabular}

Among the 14 banks, 6 ones are efficient based on the CCR model. The efficient unit set $\mathrm{E}^{*}$ contains Jiujiang Bank $(A)$, Hankou Bank $(B)$, Huishang Bank $(C)$, Tianjin Bank $(D)$, Chongqing Bank $(E)$, and ZHHR Bank $(F)$. According to Definition 2 and 3, we can compute $C(S)$ and $C(S /\{k\})$ of these banks, respectively. Table 5 shows the results of Jiujiang Bank $(A)$.

Table 5 Procedures for computing the Shapley value of Jiujiang Bank

\begin{tabular}{cccccc}
\hline$S$ & $C(S)$ & $C(S /\{k\})$ & $S$ & $C(S)$ & $C(S /\{k\})$ \\
\hline$\{A\}$ & 0.4778 & 0 & $\{A, B, C, D\}$ & 1.9254 & 1.1347 \\
$\{A, B\}$ & 0.9230 & 0.4279 & $\{A, B, C, E\}$ & 3.0088 & 2.2178 \\
$\{A, C\}$ & 1.1502 & 0.3851 & $\{A, B, C, F\}$ & 1.6593 & 0.8682 \\
$\{A, D\}$ & 0.7522 & 0.2743 & $\{A, B, D, E\}$ & 4.0401 & 3.5356 \\
$\{A, E\}$ & 0.5750 & 0.0972 & $\{A, B, D, F\}$ & 1.2392 & 0.7387 \\
$\{A, F\}$ & 0.5132 & 0.0354 & $\{A, B, E, F\}$ & 2.3632 & 1.8681 \\
$\{A, B, C\}$ & 1.6040 & 0.8130 & $\{A, C, D, E\}$ & 1.8988 & 1.1341 \\
$\{A, B, D\}$ & 1.2039 & 0.7034 & $\{A, C, D, F\}$ & 1.5269 & 0.7622 \\
$\{A, B, E\}$ & 2.3278 & 1.8327 & $\{A, C, E, F\}$ & 1.3027 & 0.5375 \\
$\{A, B, F\}$ & 0.9583 & 0.4632 & $\{A, D, E, F\}$ & 1.1989 & 0.7211 \\
$\{A, C, D\}$ & 1.4716 & 0.7069 & $\{A, B, C, D, E\}$ & 4.7118 & 3.9212 \\
$\{A, C, E\}$ & 1.2474 & 0.4823 & $\{A, B, C, D, F\}$ & 1.9806 & 1.1900 \\
$\{A, C, F\}$ & 1.2055 & 0.4404 & $\{A, B, C, E, F\}$ & 3.0641 & 2.2731 \\
$\{A, D, E\}$ & 1.1635 & 0.6857 & $\{A, B, D, E, F\}$ & 4.0754 & 3.5710 \\
$\{A, D, F\}$ & 0.7875 & 0.3097 & $\{A, C, D, E, F\}$ & 1.9541 & 1.1893 \\
$\{A, E, F\}$ & 0.6104 & 0.1325 & $\{A, B, C, D, E, F\}$ & 4.7670 & 3.9764 \\
\hline
\end{tabular}

Table 6 shows the Shapley value of each DMU based on Formula (6). Since there are six DMUs in efficient set $E^{*}$, based on the number of DMUs in the coalition $S$, the Shapley value can be decomposed into six parts. In Table $6, p=1$ represents scores obtained when $S$ contains only one DMU, $p=6$ represents scores obtained when $S$ contains all the six efficient DMUs."

The six efficient DMUs in Table 6 have different Shapley values. Therefore, our approach can discriminate efficient DMUs which can't be done by traditional CCR model. In addition, the 
efficiency of each DMU is obtained based on a common platform, which has considered all possible evaluation standards. Table 7 shows the comparison of our approach with the super efficiency model.

Table 6 Shapley values of six banks

\begin{tabular}{cccccccc}
\hline NO & $p=1$ & $p=2$ & $p=3$ & $p=4$ & $p=5$ & $p=6$ & Shapley value \\
\hline$A$ & 0.0796 & 0.0898 & 0.1002 & 0.1108 & 0.1214 & 0.1318 & 0.6335 \\
$B$ & 0.0713 & 0.1155 & 0.1785 & 0.2592 & 0.3564 & 0.4688 & 1.4497 \\
$C$ & 0.0642 & 0.0760 & 0.0882 & 0.0997 & 0.1092 & 0.1153 & 0.5525 \\
$D$ & 0.0457 & 0.0578 & 0.0888 & 0.1376 & 0.2030 & 0.2838 & 0.8168 \\
$E$ & 0.0162 & 0.0703 & 0.1431 & 0.2339 & 0.3414 & 0.4644 & 1.2692 \\
$F$ & 0.0059 & 0.0066 & 0.0072 & 0.0079 & 0.0085 & 0.0092 & 0.0453 \\
\hline
\end{tabular}

Table 7 also shows the rank of each DMU based on the super efficiency model and our approach, respectively. The last bank, Chongqing Bank $(E)$ has the same rank via the two approaches, while the first five banks have different ranks.

Table 7 Evaluation results of the super efficiency model and our approach

\begin{tabular}{cccccc}
\hline NO. & SE & Rank (SE) & Shapley value & Rank & Rank change \\
\hline$A$ & 1.4778 & 1 & 0.6335 & 4 & -3 \\
$B$ & 1.4279 & 2 & 1.4497 & 1 & +1 \\
$C$ & 1.3851 & 3 & 0.5525 & 5 & -2 \\
$D$ & 1.2743 & 4 & 0.8168 & 3 & +1 \\
$E$ & 1.0972 & 5 & 1.2692 & 2 & +3 \\
$F$ & 1.0354 & 6 & 0.0453 & 6 & 0 \\
\hline
\end{tabular}

We use Jiujiang Bank $(A)$ and Chongqing Bank $(E)$ as examples to explain the difference. The efficiency computed by super efficiency model of Jiujiang Bank $(A)$ is 1.4778 . This score is the highest among the scores of the six banks. However, the Shapley value of Jiujiang Bank $(A)$ in our model is 0.6335 , which is just the fourth higher among the six efficient DMUs. The super efficiency of Chongqing Bank $(E)$ is 1.097 while its Shapley value is 1.269. The rank of this bank in the super efficiency model is the fifth. Its rank increases to the second based on our approach.

The evaluation results of Jiujiang Bank $(A)$ and Chongqing Bank $(E)$ in the two approaches are vastly different. The difference is due to the existence of multi-standard problems in the super efficiency model. The second column in Table 6 that is, $p=1$, is computed by the super efficiency 
score and the weight of $1 / q$. Thus, the super efficiency score can be deemed as one step ahead of our model because $q$ is determined. The highest efficiency score is obtained by Jiujiang Bank $(A)$. Therefore, the rank by the second column is consistent with the result by the super efficiency model. However, when the number of subset increases to two, Jiujiang Bank $(A)$ does not obtain the highest score. On the contrary, Chongqing Bank $(E)$ has more effect on the coalition when $p>2$ (The $S$ has more than two DMUs). As a result, Chongqing Bank $(E)$ has more effect to the efficient frontier than Jiujiang Bank $(A)$. Thus, the Shapley value of Chongqing Bank $(E)$ is higher than Jiujiang Bank $(A)$.

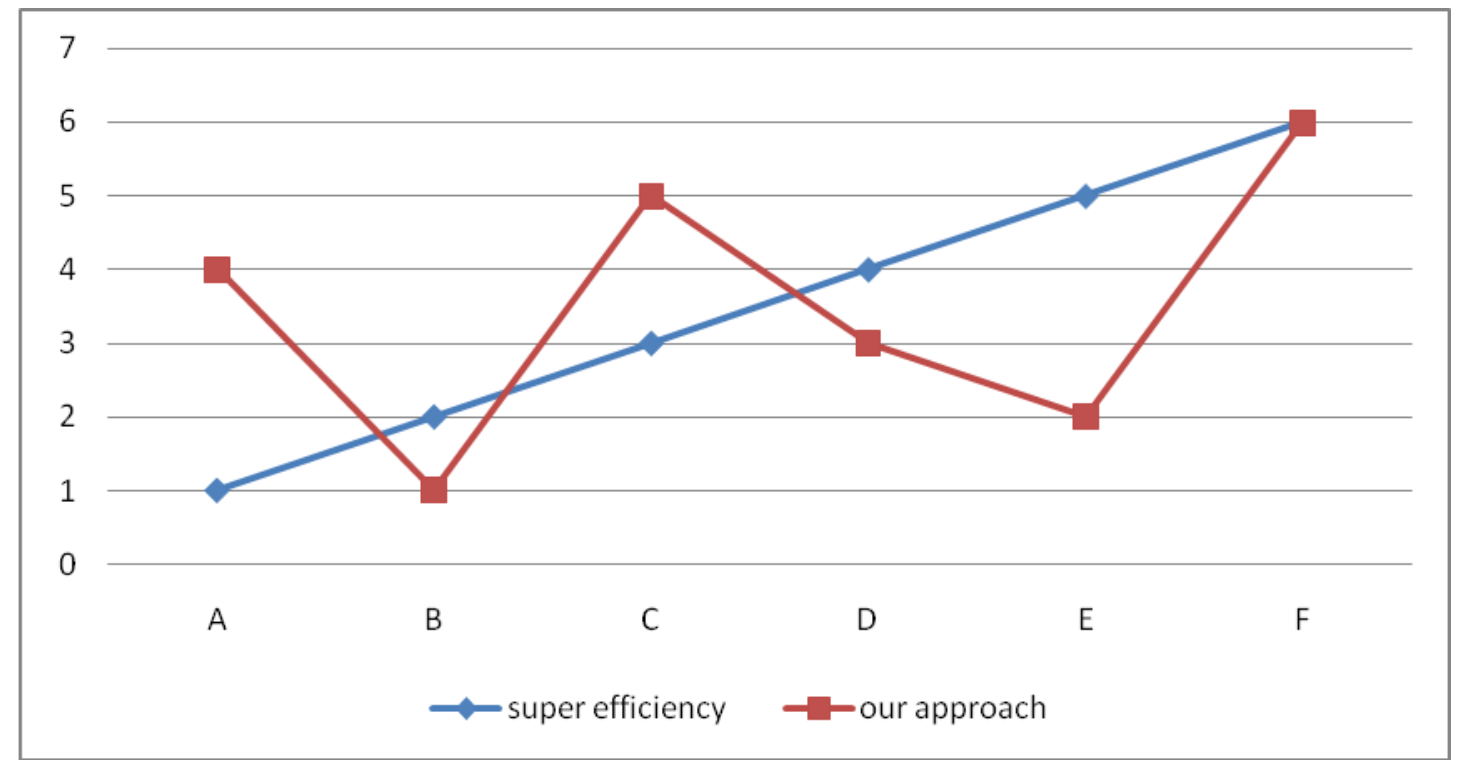

Figure 3 Rank of banks in super efficiency model and our model

\section{Extension to VRS}

This section extends the proposed approach from the constant return to scale (CRS) assumption to the VRS assumption. Due to the infeasibility of the super efficiency model based the VRS assumption (Baker \& Talluri, 1997; Seiford \& Zhu, 1999), here we use two extensions of the slack-based measure model to replace the traditional super efficiency model in the proposed approach. One is the super slack-based measure (S-SBM) model developed by Tone (2002) and the other is the joint SBM (JSBM) model. The two models are always feasible (Tone, 2002; Chen, 2013).

We first introduce the initial SBM model developed by Tone (2001) as follows. 


$$
\begin{array}{ll}
E_{f}^{S B M}(N)=\operatorname{Min} \frac{1-\frac{1}{m} \sum_{i \in M} s_{i f}^{-} / x_{i f}}{1+\frac{1}{s} \sum_{r \in H} s_{r f}^{+} / y_{r f}} \\
\text { s.t. } \quad \sum_{j \in N} \lambda_{j} x_{i j}=x_{i f}-s_{i f}^{-}, \forall i \in M \\
\quad \sum_{j \in N} \lambda_{j} y_{r j}=y_{r d}+s_{r f}^{-}, \forall r \in H \\
\quad \sum_{j \in N}^{n} \lambda_{j}=1 \\
\lambda_{j} \geq 0, s_{i f}^{-} \geq 0, s_{r f}^{-} \geq 0, \forall i, r, j
\end{array}
$$

Model (7) is a fractional programming, and can be transformed to the following linear programming via Charnes-Cooper transformation (Charnes, Cooper, \& Rhodes, 1978; Tone, 2001).

$$
\begin{array}{ll}
E_{f}^{S B M}(N)=\operatorname{Min} \quad t-\frac{1}{m} \sum_{i \in M} s_{i f}^{-} / x_{i f} \\
\text { s.t. } \quad t+\frac{1}{s} \sum_{r \in H} s_{r f}^{+} / y_{r f}=1 \\
& \sum_{j \in N} \lambda_{j} x_{i j}=t x_{i f}-s_{i f}^{-}, \forall i \in M \\
& \sum_{j \in N} \lambda_{j} y_{r j}=t y_{r d}+s_{r f}^{-}, \forall r \in H \\
& \sum_{j \in N} \lambda_{j}=1 \\
& \lambda_{j} \geq 0, s_{i f}^{-} \geq 0, s_{r f}^{-} \geq 0, \forall i, r, j
\end{array}
$$

Contrary to radial projection models, weakly efficient situations do not exist in SBM models. Therefore, the TU-game players set $E^{*}=\left\{f: E_{f}^{S B M}(N)=1\right\}$ in this section only include the strong efficient DMUs. Then, similar to the model (3) in Section 2, when a subset $S$ ( $\left.S \subseteq E^{*}\right)$ is removed from the reference set, the super efficiencies $E_{d}^{S S B M}(N / S), \forall d \in E^{*}$ can be calculated by the following S-SBM model (9). 


$$
\begin{gathered}
E_{d}^{S S B M}(N / S)=\operatorname{Min} \frac{1+\frac{1}{m} \sum_{i \in M} s_{i d}^{-} / x_{i d}}{1-\frac{1}{s} \sum_{r \in H} s_{r d}^{+} / y_{r d}} \\
\text { s.t. } \quad \sum_{j \in N / S} \lambda_{j} x_{i j}=x_{i d}+s_{i d}^{-}, \forall i \in M \\
\sum_{j \in N / S} \lambda_{j} y_{r j}=y_{r d}-s_{r d}^{-}, \forall r \in H \\
\sum_{j \in N / S} \lambda_{j}=1 \\
\lambda_{j} \geq 0, s_{i d}^{-} \geq 0, s_{r d}^{-} \geq 0, \forall i, r, j
\end{gathered}
$$

Fractional programming (9) can be transformed to the following linear programming (10).

$$
\begin{gathered}
E_{d}^{S S B M}(N / S)=\operatorname{Min} \quad t+\frac{1}{m} \sum_{i \in M} s_{i d}^{-} / x_{i d} \\
\text { s.t. } \quad t-\frac{1}{s} \sum_{r \in H} s_{r d}^{+} / y_{r d}=1 \\
\sum_{j \in N / S} \lambda_{j} x_{i j}=t x_{i d}+s_{i d}^{-}, \forall i \in M \\
\sum_{j \in N / S}^{n} \lambda_{j} y_{r j}=t y_{r d}-s_{r d}^{-}, \forall r \in H \\
\sum_{j \in N / S}^{n} \lambda_{j}=1 \\
\lambda_{j} \geq 0, s_{i d}^{-} \geq 0, s_{r d}^{-} \geq 0, \forall i, r, j
\end{gathered}
$$

Definition 4: Same as CRS models, we also define an ECP in this section, and we can also obtain the following equation.

$$
\operatorname{ECP}_{d}^{S S B M}(S)=E_{d}^{S S B M}(N / S)-1, \forall d \in E^{*}, S \subseteq E^{*}
$$

Following Tone (2002) and Chen (2013), we can easily prove: if $d \in S, \operatorname{ECP}_{d}^{S S B M}(S) \geq 0$; if $d \in E^{*} / S, \quad E_{C} P_{d}^{S S B M}(S)=0$; if $S=\varnothing$, which means no DMUs have been removed from the reference set, then $\operatorname{ECP}_{d}^{S S B M}(S)=0$.

Definition 5 Similarly, the characteristic function $C_{d}^{S S B M}(S)$ for common platform TU-game based on S-SBM model is defined as 


$$
C^{S S B M}(S)=\sum_{d \in S} E C P_{d}^{S S B M}(S)
$$

Obviously, if $S=\varnothing$, then $C_{d}^{S S B M}(S)=0$.

Theorem 2: The characteristic function $C_{d}^{S S B M}(S)$ is super-additive.

Proof. See Appendix.

From Definition 4, Definition 5, and Theorem 2, we learn that the TU-game defined in Section 4 can also be applied here. After calculating the results by S-SBM model (10), a Shapley value can be computed as the unique solution for the fairness axioms presented in Section 4.

We use the data set in Table 2 to illustrate the VRS case of the proposed approach. Results are presented in Table 8. Similarly, five DMUs are VRS-efficient, while the last three DMUs are inefficient. The $2^{\text {nd }}$ and $3^{\text {rd }}$ columns show the super efficiency and ranks obtained by S-SBM model, the $4^{\text {th }}$ and $5^{\text {th }}$ columns are the Shapley value and ranks based on the common platform approach, and the last column shows rank changes between the two approaches. It shows DMU $A$ and $D$ are ranked differently.

Table 8 Results of SBM model based on VRS assumption

\begin{tabular}{cccccc}
\hline Unit & Super efficiency & Rank (SE) & Shapley value & Rank & Rank change \\
\hline$A$ & 1.2976 & 4 & 0.7635 & 3 & -1 \\
$B$ & 2.0000 & 2 & 1.2252 & 2 & 0 \\
$C$ & 1.0667 & 5 & 0.3252 & 5 & 0 \\
$D$ & 1.6667 & 3 & 0.7221 & 4 & +1 \\
$M$ & 2.1667 & 1 & 1.3734 & 1 & 0 \\
\hline
\end{tabular}

Notably, the common platform S-SBM method can also be applied to the CRS assumption. Here we use the S-SBM model as an example to extend the proposed approach in this paper. Similarly, we can use the J-SBM (Chen, 2013) to extend it as well.

\section{Conclusion}

In this paper, we propose an approach to evaluate DMUs using a common platform to compute the efficiencies of DMUs in a comparable and equitable way. The set of all efficient DMUs is regarded as a grand coalition and each efficient unit is a player. Based on the axioms of fairness, Shapley values 
are obtained for the cooperative game. Simultaneously, we extend our method to a VRS case. As a real-world example, our approach is applied to analyze the efficiencies of 14 CCCBs. Based on our model, the Shapley values of these banks and a full ranking are given.

A limitation is that the proposed approach only calculates the efficiencies of efficient units. Inefficient DMUs, which can be evaluated in the CCR model, cannot be directly compared with the evaluations of efficient DMUs computed by our approach. In addition, when many efficient DMUs exist, the algorithm becomes complicated and requires much time to run. Another problem is that some inefficient units probably have an effect on efficient DMUs. This effect is an issue because the cooperative game may not be super-additive when considering all DMUs in the DMU set as players. For future research, the proposed approach may be extended to compute the efficiency of inefficient unit in a similar way. Regarding all units in the DMU set as a coalition is also recommended.

\section{Acknowledgment}

The authors thank Editor Professor Dyson, an associate editor, and three reviewers. The authors thank Professor Joe Zhu for his helpful comments and suggestions, which significantly improved the paper. The authors also thank Mr. Xiao Tang for his contribution on the initial version of this paper. This research was supported by the Youth Innovation Promotion Association of Chinese Academy of Sciences, the National Natural Science Foundation of China (Grant No. 71271196), the Science Fund for Creative Research Groups of the National Natural Science Foundation of China (Grant No. 71121061), the Fund for International Cooperation and Exchange of the National Natural Science Foundation of China (Grant No. 71110107024), and the National Science Foundation of China for Distinguished Youth Scholars (Grant No. 71225002). 


\section{References}

Adler, N., Friedman, L., \& Sinuany-Stern, Z. (2002). Review of ranking methods in the data envelopment analysis context. European Journal of Operational Research, 140(2), 249-265.

Alcaraz, J., Ramón, N., Ruiz, J. (2013). Ranking ranges in cross-efficiency evaluations. European Journal of Operational Research, 226(3), 516-521.

Andersen, P., \& Petersen, N. C. (1993). A procedure for ranking efficient units in data envelopment analysis. Management science, 39(10), 1261-1264.

Angulo-Meza, L., \& Lins, M. P. E. (2002). Review of methods for increasing discrimination in data envelopment analysis. Annals of Operations Research, 116(1-4), 225-242.

Avkiran, N. K. (2011). Association of DEA super-efficiency estimates with financial ratios: Investigating the case for Chinese banks. Omega, 39(3), 323-334.

Baker, R. C., \& Talluri, S. (1997). A closer look at the use of data envelopment analysis for technology selection. Computers \& Industrial Engineering, 32(1), 101-108.

Banker, R. D., \& Chang, H. (2006). The super-efficiency procedure for outlier identification, not for ranking efficient units. European Journal of Operational Research, 175(2), 1311-1320.

Banker, R. D., Chang, H., \& Zheng Z. (2015). On the use of super-efficiency procedures for ranking efficient units and identifying outliers. Annals of Operations Research, 116(1-4), 1-15.

Banker, R. D., Charnes, A., \& Cooper, W. W. (1984). Some models for estimating technical and scale inefficiencies in data envelopment analysis. Management science, 30(9), 1078-1092.

Borrero, D. V., Hinojosa, M. A., \& Mármol, A. M. (2016). DEA production games and Owen allocations. European Journal of Operational Research, 252(3), 921-930.

Charnes, A., Cooper, W. W., \& Rhodes, E. (1978). Measuring the efficiency of decision making units. European journal of operational research, 2(6), 429-444.

Chen, C. M. (2013). Super efficiencies or super inefficiencies? Insights from a joint computation model for slacks-based measures in DEA. European Journal of Operational Research.226, 258-267 
Chen, J. X., \& Deng, M. (2011). A cross-dependence based ranking system for efficient and inefficient units in DEA. Expert Systems with Applications, 38(8), 9648-9655.

Chen, Y., \& Liang, L. (2011). Super-efficiency DEA in the presence of infeasibility: One model approach. European Journal of Operational Research, 213(1), 359-360.

Cook, W. D., Liang, L., Zha, Y., \& Zhu, J. (2008). A modified super-efficiency DEA model for infeasibility. Journal of the Operational Research Society, 60(2), 276-281.

Cook, W., Roll, Y., \& Kazakov, A. (1990). DEA model for measuring the relative efficiencies of highway maintenance patrols. INFOR, 28 (2), 113-124.

Cooper, W. W., Seiford, L. M., \& Tone, K. (2007). Data envelopment analysis: a comprehensive text with models, applications, references and DEA-solver software. Springer Science, Business Media. Cooper, W. W., Seiford, L. M., \& Zhu, J. (2011). Data envelopment analysis: History, models, and interpretations. Handbook on data envelopment analysis. Springer US.

Debreu, G. (1951). The coefficient of resource utilization. Econometrica: Journal of the Econometric Society, 273-292.

Du, J., Liang, L., Chen, Y., Cook, W. D., \& Zhu, J. (2011). A bargaining game model for measuring performance of two-stage network structures. European Journal of Operational Research, 210(2), 390-397.

Düzakın, E., \& Düzakın, H. (2007). Measuring the performance of manufacturing firms with super slacks based model of data envelopment analysis: An application of 500 major industrial enterprises in Turkey. European Journal of Operational Research, 182(3), 1412-1432.

Doyle, J., \& Green, R. (1994). Efficiency and cross-efficiency in DEA: Derivations, meanings and uses. Journal of the Operational Research Society 43, 567-578.

Farrell, M. J. (1957). The measurement of productive efficiency. Journal of the Royal Statistical Society. Series A (General), 120(3), 253-290.

Hibiki, N., \& Sueyoshi, T. (1999). DEA sensitivity analysis by changing a reference set: regional contribution to Japanese industrial development. Omega, 27(2), 139-153. 
Hua, Z., Bian, Y., \& Liang, L. (2007). Eco-efficiency analysis of paper mills along the Huai River: an extended DEA approach. Omega, 35(5), 578-587.

Jahanshahloo, G. R., Junior, H. V., Lotfi, F. H., \& Akbarian, D. (2007). A new DEA ranking system based on changing the reference set. European Journal of Operational Research, 181(1), 331-337.

Johnson, A. L., \& McGinnis, L. F. (2009). The hyperbolic-oriented efficiency measure as a remedy to infeasibility of super efficiency models. Journal of the Operational Research Society, 60(11), $1511-1517$.

Kao, C., \& Hung, H. T. 2005. Data envelopment analysis with common weights: the compromise solution approach. Journal of the Operational Research Society, 56(10), 1196-1203.

Kao, C. 2010. Malmquist productivity index based on common-weights DEA: The case of Taiwan forests after reorganization. Omega, 38(6), 484-491.

Lee, H. S., Chu, C. W., \& Zhu, J. (2011). Super-efficiency DEA in the presence of infeasibility. European Journal of Operational Research, 212(1), 141-147.

Lee, H. S., \& Zhu, J. (2012). Super-efficiency infeasibility and zero data in DEA. European Journal of Operational Research, 216(2), 429-433.

Li, Y., Chen, Y., Liang, L., \& Xie, J. (2012). DEA models for extended two-stage network structures. Omega, 40(5), 611-618.

Li, Y., \& Liang, L. (2010). A Shapley value index on the importance of variables in DEA models. Expert Systems with Applications, 37(9), 6287-6292.

Li, Y., Yang, F., Liang, L., \& Hua, Z. (2009). Allocating the fixed cost as a complement of other cost inputs: A DEA approach. European Journal of Operational Research, 197(1), 389-401.

Liang, L., Cook, W. D., \& Zhu, J. (2008). DEA models for two - stage processes: Game approach and efficiency decomposition. Naval Research Logistics (NRL), 55(7), 643-653.

Liang, L., Wu, J., Cook, W. D., \& Zhu, J. (2008). The DEA game cross-efficiency model and its Nash equilibrium. Operations Research, 56(5), 1278-1288. 
Liang, L., Wu, J., Cook, W. D., \& Zhu, J. (2008). Alternative secondary goals in DEA cross-efficiency evaluation. International Journal of Production Economics, 113(2), 1025-1030

Lim, S., \& Zhu, J. (2013). Incorporating Performance Measures with Target Levels in Data Envelopment Analysis. European Journal of Operational Research, 230(3), 634-642.

Lozano, S. (2012). Information sharing in DEA: A cooperative game theory approach. European Journal of Operational Research, 222(3), 558-565.

Lozano, S. (2013). DEA production games. European Journal of Operational Research. 231, 405413.

Lozano, S., Hinojosa, M. A., \& Mármol, A. M. (2015). Set-valued DEA production games. Omega, 52, 92-100.

Myerson, R. B. (1980). Conference structures and fair allocation rules. International Journal of Game Theory, 9(3), 169-182.

Nakabayashi, K., \& Tone, K. (2006). Egoist's dilemma: a DEA game. Omega, 34(2), 135-148.

Osborne, M. J., \&Rubinstein, A. (1994). A course in game theory. Cambridge, Mass.: MIT Press.

Pulina, M., Detotto, C., \& Paba, A. (2010). An investigation into the relationship between size and efficiency of the Italian hospitality sector: A window DEA approach. European Journal of Operational Research, 204(3), 613-620.

Roll, Y., Cook, W. D., \& Golany, B. 1991. Controlling factor weights in data envelopment analysis. IIE transactions, 1991, 23(1), 2-9.

Ruiz, J. L. (2013). Cross-efficiency evaluation with directional distance functions. European Journal of Operational Research, 228(1), 181-189.

Salo, A., \& Punkka, A. (2011). Ranking intervals and dominance relations for ratio-based efficiency analysis. Management Science, 57(1), 200-214.

Scheel, H. (2001). Undesirable outputs in efficiency valuations. European Journal of Operational Research, 132: 400-410. 
Seiford, L. M., \& Zhu, J. (1999). Infeasibility of super-efficiency data envelopment analysis models. Infor, 37(2), 174-187.

Seiford, L. M., \& Zhu, J. (2002).Modeling undesirable factors in efficiency evaluation. European Journal of Operational Research,142: 16-20.

Shapley, L. S. (1953). A value for N-person games. Contribution to the Theory of Games, Vol. 2. Princeton University Press, Princeton, NJ: 307-317.

Shwartza, M., Burgess, J., \& Zhu, J. (2016). A DEA based composite measure of quality and its associated data uncertainty interval for health care provider profiling and pay-for-performance. European Journal of Operational Research, 253 (2): 489-502.

Staub, R. B., da Silva e Souza, G., \& Tabak, B. M. (2010). Evolution of bank efficiency in Brazil: A DEA approach. European journal of operational research, 202(1), 204-213.

Tone, K.(2001). A slacks-based measure of efficiency in data envelopment analysis. European Journal of Operational Research 130 (3), 498-509.

Tone, K.(2002). A slacks-based measure of super-efficiency in data envelopment analysis. European Journal of Operational Research 143 (1), 32-41.

Wanke, P., Barros, C.P., \& Emrouznejad. (2016). Assessing productive efficiency of banks using integrated Fuzzy-DEA and bootstrapping: A case of Mozambican banks. European Journal of Operational Research 249 (1), 378-389.

Wu, H., Du, S., Liang, L., \& Zhou, Y. (2013). A DEA-based approach for fair reduction and reallocation of emission permits. Mathematical and Computer Modelling, 58(5), 1095-1101.

Wu, J., Chu, J., Sun, J., \& Zhu, Q. (2016). DEA cross-efficiency evaluation based on Pareto improvement. European Journal of Operational Research, 248(2), 571-579.

Wu, J., Liang, L., \& Yang, F. (2009). Determination of the weights for the ultimate cross efficiency using Shapley value in cooperative game. Expert Systems with Applications, 36(1), 872-876.

Wu, J., Liang, L., \& Chen, Y. (2009). DEA game cross-efficiency approach to Olympic rankings. Omega, 37(4), 909-918. 
Wu, J., Liang, L., Yang, F., \& Yan, H. (2009). Bargaining game model in the evaluation of decision making units. Expert Systems with Applications, 36(3), 4357-4362.

Wu, J., Zhu, Q., Cook, W. D., \& Zhu, J. (2016). Best cooperative partner selection and input resource reallocation using DEA. Journal of the Operational Research Society, Forthcoming.

Zha, Y., \& Liang, L. (2010). Two-stage cooperation model with input freely distributed among the stages. European Journal of Operational Research, 205(2), 332-338.

Zhou Z., Sun L., Yang W., Liu W., \& Ma C. (2013). A bargaining game model for efficiency decomposition in the centralized model of two-stage systems. Computers \& Industrial Engineering, 64,103-108

Zhu, J. (2004). A buyer-seller game model for selection and negotiation of purchasing bids: extensions and new models. European Journal of Operational Research, 154(1), 150-156. 


\section{Appendix}

Lemma 1: $E_{d}(N /\{k\}) \geq E_{d}(N), \forall k, d \in E^{*}$

Proof: If $k=d$, then $E_{d}(N /\{k\})$ denotes the super efficiency of $d$ and $E_{d}(N /\{k\}) \geq E_{d}(N), \forall k \in E^{*}$ (Lee, Chu, \& Zhu, 2011). If $k \neq d$, then $E_{d}(N /\{k\})=E_{d}(N)=1$ as $k$ and $d$ are both efficient DMUs.

Lemma 2: $\operatorname{ECP}_{d}(S) \geq 0, \forall d \in E^{*}, S \subseteq E^{*}$.

Proof. $E_{d}(N)=1$, If $d \in S$ and $E_{d}(N / S) \geq 1$, then based on Lemma 1 , we have $E C P_{d}(S)=\frac{E_{d}(N / S)}{E_{d}(N)}-1=E_{d}(N / S)-1 \geq 0 \quad$. If $\quad d \notin S \quad$ and $\quad E_{d}(N / S)=1 \quad, \quad$ then $E C P_{d}(S)=\frac{E_{d}(N / S)}{E_{d}(N)}-1=E_{d}(N / S)-1=0$.

Lemma 3: $E_{d}(N / S) \geq E_{d}(N), \forall d \in E^{*}, S \subseteq E^{*}$

Proof. Based on Lemma 2, we have $E C P_{d}(S) \geq 0, \forall d \in E^{*}, S \subseteq E^{*}, \quad E C P_{d}(S)=\frac{E_{d}(N / S)}{E_{d}(N)}-1 \geq 0$, $\frac{E_{d}(N / S)}{E_{d}(N)} \geq 1$. Therefore, $E_{d}(N / S) \geq E_{d}(N)$

Lemma 4: $E C P_{d}\left(S_{1} \cup S_{2}\right) \geq E C P_{d}\left(S_{1}\right)+E C P_{d}\left(S_{2}\right), \forall d \in E^{*}, S_{1} \subseteq E^{*}, S_{2} \subseteq E^{*}, S_{1} \cap S_{2}=\varnothing$.

Proof. To prove Lemma 4, we need to prove

$$
\frac{E_{d}\left(N /\left(S_{1} \cup S_{2}\right)\right)}{E_{d}(N)}+1 \geq \frac{E_{d}\left(N / S_{1}\right)}{E_{d}(N)}+\frac{E_{d}\left(N / S_{2}\right)}{E_{d}(N)},
$$

which means $E_{d}\left(N /\left(S_{1} \cup S_{2}\right)\right)+E_{d}(N) \geq E_{d}\left(N / S_{1}\right)+E_{d}\left(N / S_{2}\right)$.

Given that $E_{d}(N)=1$, then we have $E_{d}\left(N /\left(S_{1} \cup S_{2}\right)\right)+1 \geq E_{d}\left(N / S_{1}\right)+E_{d}\left(N / S_{2}\right)$.

If $d \in S_{1}$, then $E_{d}(N)=E_{d}\left(N / S_{2}\right)=1 ;$ and based on Lemma 3 , we have $E_{d}\left(N /\left(S_{1} \cup S_{2}\right)\right) \geq E_{d}\left(N / S_{1}\right)$. 
If $d \in S_{2}$, then $E_{d}(N)=E_{d}\left(N / S_{1}\right)=1 ;$ and based on Lemma 3, we have $E_{d}\left(N /\left(S_{1} \cup S_{2}\right)\right) \geq E_{d}\left(N / S_{2}\right)$.

If $d \notin S_{1}$ and $d \notin S_{2}$, then $E_{d}(N)=E_{d}\left(N / S_{1}\right)=E_{d}\left(N / S_{2}\right)=E_{d}\left(N /\left(S_{1} \cup S_{2}\right)\right)=1$.

Therefore, $E_{C} P_{d}\left(S_{1} \cup S_{2}\right) \geq E C P_{d}\left(S_{1}\right)+E C P_{d}\left(S_{2}\right), \forall d \in E^{*}, S_{1} \subseteq E^{*}, S_{2} \subseteq E^{*}, S_{1} \cap S_{2}=\varnothing$.

Theorem 1: The characteristic function $C$ is super-additive. In particular, for any $S_{1} \subseteq E^{*}$ and $S_{2} \subseteq E^{*}$ with $S_{1} \cap S_{2}=\varnothing$, we obtain

$$
C\left(S_{1} \cup S_{2}\right) \geq C\left(S_{1}\right)+C\left(S_{2}\right)
$$

Proof. Based on Lemma 4, we obtain

$$
\begin{aligned}
E C P_{d}\left(S_{1} \cup S_{2}\right) \geq & E C P_{d}\left(S_{1}\right)+E C P_{d}\left(S_{2}\right), \forall d \in E^{*}, S_{1} \subset E^{*}, S_{2} \subset E^{*}, S_{1} \cap S_{2}=\varnothing \text { and } \\
& \sum_{d \in S_{1} \cup S_{2}} E C P_{d}\left(S_{1} \cup S_{2}\right) \geq \sum_{d \in S_{1} \cup S_{2}} E C P_{d}\left(S_{1}\right)+\sum_{d \in S_{1} \cup S_{2}} E C P_{d}\left(S_{2}\right) .
\end{aligned}
$$

Thus, we can say that the characteristic function $C$ is super-additive.

Theorem 2: The characteristic function $C_{d}^{S S B M}(S)$ is super-additive.

\section{Proof.}

To prove that $C_{d}^{S S B M}(S)$ is super-additive is equivalent to proving that, for any two sets $S_{1} \subseteq E^{*}$, $S_{2} \subseteq E^{*}$ and $S_{1} \cap S_{2}=\varnothing$, there is

$$
C^{S S B M}\left(S_{1} \cup S_{2}\right) \geq C^{S S B M}\left(S_{1}\right)+C^{S S B M}\left(S_{2}\right)
$$

As defined in Definition 4 and Definition 5, we obtain

$$
\begin{aligned}
& C^{S S B M}\left(S_{1} \cup S_{2}\right)=\sum_{d \in S_{1} \cup S_{2}} E C P_{d}^{S S B M}\left(S_{1} \cup S_{2}\right)=\sum_{d \in S_{1} \cup S_{2}} E_{d}^{S S B M}\left(N /\left(S_{1} \cup S_{2}\right)\right)-p_{3}, \\
& C^{S S B M}\left(S_{1}\right)=\sum_{d \in S_{1}} E C P_{d}^{S S B M}\left(S_{1}\right)=\sum_{d \in S_{1}} E_{d}^{S S B M}\left(N / S_{1}\right)-p_{1} \\
& C^{S S B M}\left(S_{2}\right)=\sum_{d \in S_{2}} E C P_{d}^{S S B M}\left(S_{2}\right)=\sum_{d \in S_{2}} E_{d}^{S S B M}\left(N / S_{2}\right)-p_{2}
\end{aligned}
$$

where $p_{3}=p_{1}+p_{2}$. 
The formula $\sum_{d \in S_{1} \cup S_{2}} E_{d}^{S S B M}\left(N /\left(S_{1} \cup S_{2}\right)\right)$ can be decomposed as $\sum_{d \in S_{1}} E_{d}^{S S B M}\left(N /\left(S_{1} \cup S_{2}\right)\right)+\sum_{d \in S_{2}} E_{d}^{S S B M}\left(N /\left(S_{1} \cup S_{2}\right)\right)$

Comparing $E_{d}^{S S B M}\left(N /\left(S_{1} \cup S_{2}\right)\right)$ with $E_{d}^{S S B M}\left(S_{1}\right)$ for arbitrary $d \in S_{1}$, we learn that the reference set $N /\left(S_{1} \cup S_{2}\right) \subseteq N / S_{1}$, which means moving the frontier of $N /\left(S_{1} \cup S_{2}\right)$ to $N / S_{1}$, would increase inputs or decrease outputs. Then, we learn that the optimal slacks of model (11) satisfy the following inequalities: $s_{i d}^{-}\left(N /\left(S_{1} \cup S_{2}\right)\right) \leq s_{i d}^{-}\left(N / S_{1}\right)$ and $s_{r d}^{+}\left(N /\left(S_{1} \cup S_{2}\right)\right) \leq s_{r d}^{+}\left(N / S_{1}\right)$ for arbitrary $\quad r \in H \quad$ and $i \in M$. Then, we obtain $E_{d}^{S S B M}\left(N /\left(S_{1} \cup S_{2}\right)\right) \geq E_{d}^{S S B M}\left(N / S_{1}\right), \forall d \in S_{1}$.

Similarly, we can obtain $E_{d}^{S S B M}\left(N /\left(S_{1} \cup S_{2}\right)\right) \geq E_{d}^{S S B M}\left(N / S_{2}\right), \forall d \in S_{2}$.

Finally, we obtain $\sum_{d \in S_{1} \cup S_{2}} E_{d}^{S S B M}\left(N /\left(S_{1} \cup S_{2}\right)\right) \geq \sum_{d \in S_{1}} E_{d}^{S S B M}\left(N / S_{1}\right)+\sum_{d \in S_{2}} E_{d}^{S S B M}\left(N / S_{1}\right)$. 